\title{
Exceptionally long-range haplotypes in Plasmodium falciparum chromosome 6 maintained in an endemic African population
}

\author{
Alfred Amambua-Ngwa1*, Bakary Danso ', Archibald Worwui', Sukai Ceesay', Nwakanma Davies', \\ David Jeffries ${ }^{1}$, Umberto D'Alessandro ${ }^{1,2}$ and David Conway ${ }^{2}$
}

\begin{abstract}
Background: Previous genome-wide analyses of single nucleotide variation in Plasmodium falciparum identified evidence of an extended haplotype region on chromosome 6 in West Africa, suggesting recent positive selection. Such a pattern is not seen in samples from East Africa or South East Asia, so it could be marking a selective process specific to West Africa. Analyses of the haplotype structure in samples taken at different times could give clues to possible causes of selection.
\end{abstract}

Methods: This study investigates chromosome 6 extended haplotypes in The Gambia by analysing alleles at multiple microsatellite loci using genome sequence data previously obtained from clinical isolates collected in 2008, followed by genotyping of 13 loci in 439 isolates from 1984, 1991, 2008 and 2014. Temporal changes in haplotype structure and frequencies were determined.

Results: A region of high linkage disequilibrium spanning over 170 kilobases (kb) was identified with both NGS and laboratory determined microsatellite alleles. Multiple long haplotypes were found in all temporal populations from The Gambia. Two of the haplotypes were detected in samples from 1984 and 1991. The frequency of long-range haplotypes increased in 2008 and 2014 populations. There was higher Fst between older and more recent populations at loci in proximity to genes involved in drug metabolism pathways.

Conclusions: The occurrence of several long haplotypes at intermediate frequencies suggests an unusual mode of selection in chromosome 6, possibly combined with recombination suppression on specific haplotypes. Such selection apparently occurred before the emergence of known anti-malarial drug resistance alleles, and could be due to effects of other drugs or unknown processes that have long been operating in this endemic region.

Keywords: Malaria, Microsatellites, Selective sweep, Haplotypes, Drug resistance

\section{Background}

Adaptation of malaria parasites to various challenges, including human immune responses, drugs and other interventions has left genetic signatures in the Plasmodium falciparum genome [1]. These can be detected with polymorphic genetic markers showing reduced variability, increased linkage disequilibrium, extended

\footnotetext{
*Correspondence: angwa@mrc.gm

${ }^{1}$ Medical Research Council, Gambia Unit, Atlantic Road, Fajara, P.O. Box 273, Banjul, The Gambia

Full list of author information is available at the end of the article
}

haplotypes, excess of rare alleles or high frequencies of derived alleles at loci close to the target of selection [2, 3]. Anti-malarial drugs in particular exert a strong positive selection on the genome and several of the strongest signatures of directional selection are linked to drug resistance genes $[4,5]$. Apart from these, a number of other P. falciparum genomic loci show evidence of positive selection in endemic populations [6, 7]. Some signatures of selection are apparent only in specific endemic populations, including an extensive selective sweep on chromosome 6 that was first described by analysing single nucleotide polymorphisms (SNPs) in parasites from 
Senegal and The Gambia in West Africa [6]. The evidence of extended haplotype homozygosity in chromosome 6 appears strong in West African parasite populations but weak or absent elsewhere [6, 8-11]. The size of the chromosomal region affected may be up to $\sim 300 \mathrm{~kb}$, and none of the $\sim 60$ genes in this region is a known direct target of selection from anti-malarial drugs. This result was obtained with SNP markers, but analysis of microsatellites may offer additional information due to their higher levels of polymorphism [12-14]. Microsatellite approaches are also attractive here because they require technology that is more accessible to low and medium income country labs. This allows for local targeted analysis of sweeps following initial scans with high density SNPs, which requires computational capacity that is limited in most of sub-Saharan Africa.

This study focuses on characterising the structure of $P$. falciparum chromosome 6 extended haplotypes around the selective signature in The Gambian population. This population benefits from prior knowledge of selective signatures from SNP studies, availability of whole genome short read sequence data for mining other polymorphisms such as microsatellites, and archived samples from the period prior to widespread chloroquine resistance. Microsatellite polymorphisms within the chromosome 6 region were targeted for this temporal analysis thanks to their abundance and sensitivity in determining haplotype structure around selective sweeps $[14,15]$. The analysis of sequence data enabled the design of new laboratory genotyping assays, which focused on a set of 13 microsatellite loci across the region of extended haplotype structure.

\section{Methods}

\section{Plasmodium falciparum isolates}

This study analysed four sets of $P$. falciparum isolates from the Gambia. The first two comprises 56 and 67 isolates collected in 1984 and 1991 respectively around Farafenni, located in the middle of the country $[4,26]$. During this period, chloroquine was still efficacious against most infections and resistance alleles were rare. The third population includes 166 isolates from the Greater Banjul area, in the west of the country, collected in 2008 when malaria transmission had decreased substantially and the first line treatment for malaria had been changed from sulfadoxine-pyrimethamine (SP) to artemether-lumefantrine (AL) [27]. The fourth was a recent population sample of 150 isolates collected in 2014 transmission season from the Greater Banjul area in the West, as well as Basse in the east of the country. Of the isolates collected in 2008, 76 had whole genome short sequence reads for mining microsatellite polymorphisms [16]. Single nucleotide polymorphisms and signatures of selection in this population has been described in a number of previous studies $[4,7,16]$.

\section{Microsatellite scoring across P. falciparum chromosome 6 using Illumina short-read sequence data}

Microsatellite markers across chromosome 6 of $P$. falciparum were determined from Illumina generated whole genome short sequence read data of the 68 isolates collected in 2008 as described previously [28]. For microsatellite identification and genotyping, short sequence reads were mapped against the $P$. falciparum 3D7 reference chromosome 6 sequences (version3, October 2012 release) to generate sequence alignment files (Bam) as previously described. Bam files were then screened for microsatellites against a reference library indexing all possible microsatellite repeats in the Pf3D7 reference chromosome 6 sequence created with the tandem repeat finder software (trf) and SciRoKo for perfect repeats $[29,30]$. Algorithms for calling microsatellite alleles are as described for the programs RepeatSeq and Genotan [31, 32]. Quality settings for calling microsatellite genotypes incorporated a short read quality score of Q30 and read coverage across the microsatellite of $5 \mathrm{X}$ spanning a unique region bordering both sides of the locus. Allele calls were corrected to lengths reflective of the repeat unit assuming a stepwise mutation model in Microsatellite Analyzer package [33]. Perfect repeat tracts with number of repeats from 14 to 63 were chosen for further analysis. The final dataset employed included loci that were polymorphic and with less than $30 \%$ missing calls. The objective was to identify a set of polymorphic markers from Illumina short sequence reads and select a subset for further analysis by PCR fragment sizing following amplification of DNA from the target populations.

\section{Selected chromosome 6 microsatellite loci for laboratory genotyping}

Microsatellite loci employed in retrospective analysis of chromosome 6 selective sweep were selected from polymorphic loci determined from short read sequence data analysed for chromosome 6 as described above. These loci were perfect microsatellites, having at least two alleles in the Gambian population and included di- to hexa-nucleotide repeats. These were physically spaced at an average distance of $\sim 15 \mathrm{~kb}$ across a $179.5 \mathrm{~kb}$ region, between positions 1069 and $1249 \mathrm{~kb}$ of the chromosome. Primer pairs were designed automatically for flanking sequences of each unique microsatellite locus with Primer3 software implemented in BatchPrimer using the Pf3D7 reference sequence as a template (Additional file 1: Targeted_13_ssr_stats). Primers for amplification and fragment labelling with 6FAM, TET, HEX and TAMRA dyes were commercially synthesized (Metabion). 


\section{PCR amplification and capillary electrophoresis}

For each sample, DNA was obtained from whole blood aliquot using the QiAmp DNA extraction kit. Amplification of targeted microsatellite loci followed a two round PCR reaction in which the first round was a multiplex for up to 3 loci in a $10 \mu \mathrm{L}$ reaction mix with $0.2 \mu \mathrm{M}$ of each outer primer pair, $1 \mu \mathrm{L}$ of DNA and $1 \mathrm{X}$ PCR multiplex mix (Qiagen). Each amplification batch included the reference $P$. falciparum 3D7 DNA as a positive control and nuclease free water as a negative control. Thermocycling was achieved with a touchdown PCR protocol on a Q-cycler (Quantarus); initial denaturation at $95^{\circ} \mathrm{C}$ for $5 \mathrm{~min}$, touchdown from 65 to $55^{\circ} \mathrm{C}$ at $1{ }^{\circ} \mathrm{C} /$ cycle followed by 25 cycles of $95^{\circ} \mathrm{C}$ for $30 \mathrm{~s}, 54^{\circ} \mathrm{C}$ for $1 \mathrm{~min}, 72^{\circ} \mathrm{C}$ for $30 \mathrm{~s}$. Final elongation was at $68{ }^{\circ} \mathrm{C}$ for $30 \mathrm{~min}$. First round PCR reactions were used immediately for second rounded amplification with labelled primers or stored at $4{ }^{\circ} \mathrm{C}$ until needed. Second round PCR reactions were run separately for each locus using $1 \mu \mathrm{L}$ of first round PCR product, $150 \mathrm{nM}$ of each primer and $1 \mathrm{X}$ of MyTaq amplification mix (Bioline) in a $10 \mu \mathrm{L}$ reaction. Amplification conditions were initial denaturation at $95^{\circ} \mathrm{C}$ for $30 \mathrm{~s}, 25$ cycles of $95{ }^{\circ} \mathrm{C}$ for $30 \mathrm{~s}, 54{ }^{\circ} \mathrm{C}$ for $30 \mathrm{~s}, 68^{\circ} \mathrm{C}$ for $30 \mathrm{~s}$. Final elongation was at $68{ }^{\circ} \mathrm{C}$ for $20 \mathrm{~min}$. For capillary electrophoresis, round $2 \mathrm{PCR}$ products were constituted at 1:50 dilution in $10 \mu \mathrm{L}$ assay mix of HIDI formamide with GeneScan $^{\text {TM }} 600$ LIZ $^{\circledR}$ size standard. This was denatured by heating at $95{ }^{\circ} \mathrm{C}$ for $3 \mathrm{~min}$ and immediately cooled on ice. Labelled microsatellite fragments were separated on 3130xL DNA analyser. DNA fragment analysis traces for loci were processed in GeneMapper 4.1 and allele sizes were called using GeneMarker 1.1. Allele sizes were checked for error and binned using Tandem 2 software, which corrects for deviations in expected fragment size based on repeat unit length of the target microsatellite. Binned genotypes were employed in determining pairwise haplotype frequencies and Linkage disequilibrium between contiguous loci using PowerMarker Version 3.5 and Midas. Fragment sizes for SSR1, SSR9 and SSR15 were correlated with illumina determined repeat length genotypes (Additional file 2).

\section{Population genetic analysis of chromosome 6 microsatellite loci}

The allele frequencies, allelic richness, variability, expected heterozygosity, and Weir and Cockerham's Fst for binned microsatellite were calculated with the heirfstat R package. Virtual heterozygosity (HE) was used to measure the overall genetic diversity at each locus. It is defined as, $\mathrm{n} /(\mathrm{n}-1)\left(1-\Sigma \mathrm{pi}^{2}\right)$, where $\mathrm{n}$ is the number of isolates analysed and pi is the frequency of the $\mathrm{i}$-th allele in the population. To test which microsatellite loci showed population variation that deviated from neutral expectations, simulations of the distribution of pairwise Fst coefficient were carried out in Bayescan 2.1. Bayescan uses posterior probabilities to control for False Discovery rates (FDR), which is the proportion of false positives among candidate outlier loci. Simulations were performed at default settings; 100,000 iterations, 5000 pilot runs and 50,000 burn-in length. P values (one-sided) were estimated from 10,000 permutations of population assuming a panmictic null hypothesis. The FDR expressed as $q$ value (FDR analogue of $p$ value defined under multiple testing) was plotted against Fst per selected loci using Bayescan plot code in R to detect and list outlier loci at a $5 \%$ FDR. Unique multilocus genotypes were determined using $\mathrm{R}$ allele Match unique package with a maximum mismatch setting at 3 out of 13 loci following mismatch and multiple match minimization. Partially matched haplotypes were further analysed with Haplotype Analysis 4.05. Visualization of the evolution of frequency of the common long range haplotype was done with the REHH package in R. For this, the loci with the lowest and highest Fst values within the window of high linkage disequilibrium were chosen as focal sites for determining the extent of haplotypes around them. To allow for REHH analysis, the fragment sizes were converted into a binary format in which '2' represented the dominant allele while ' 1 ' was for any other fragment length at this locus. Analysis and plotting were as described in the REHH manual.

\section{Results}

\section{Diversity of chromosome 6 microsatellite loci derived} from short sequence reads of $P$. falciparum

The number of repeats for 775 polymorphic microsatellite loci in chromosome 6 of P. falciparum were successfully scored from Illumina short read sequences of 54 out of 68 clinical isolates collected in 2008. The mean virtual heterozygosity for the 13 microsatellite loci was 0.47 . Pairwise linkage disequilibrium, expressed as mean $r^{2}$ values between all pairs of loci within a window of $10 \mathrm{~kb}$ containing at least 5 loci, ranged from 0 to 0.3 . The values were significantly higher towards the $3^{\prime}$ end of the chromosome, mapping between positions $1100-1300 \mathrm{~kb}$ with a peak at $\sim 1200 \mathrm{~kb}$ (Fig. 1a). Mean pairwise haplotype frequencies between loci in $10 \mathrm{~kb}$ windows across the chromosome showed a narrower peak of $\sim 100 \mathrm{~kb}$ (1150$1250 \mathrm{~kb}$ ) at this region on the chromosome (Additional file 3).

Long-range haplotypes spanning $184 \mathrm{~kb}$ were identified with microsatellite genotypes for 28 polymorphic loci across the region of peak LD for 54 isolates (Fig. 1b). These long-range haplotypes shared by at least $2-8$ isolates were represented in 28 isolates (50\%). Following allele matching and haplotype extension from 12 loci 
a

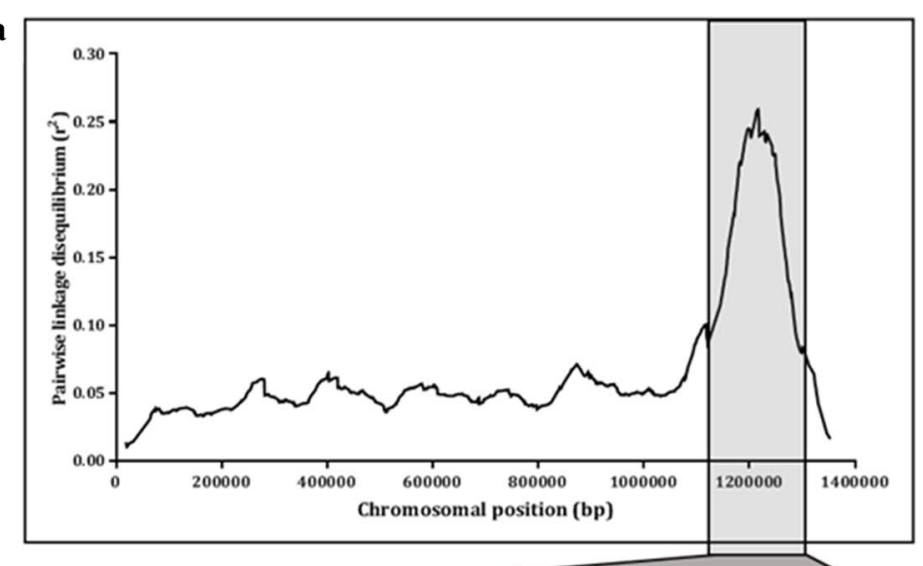

b

\begin{tabular}{|c|c|c|c|c|c|c|c|c|c|c|c|c|c|c|c|c|c|c|c|c|c|c|c|c|c|c|c|c|c|c|}
\hline & & $\$$ & 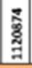 & 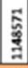 & 莺 & 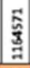 & 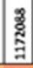 & \begin{tabular}{l}
$\widehat{్}$ \\
\multirow{z}{*}{}
\end{tabular} & 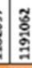 & & & & & & & 율 & 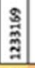 & $\begin{array}{l}\widetilde{\$} \\
\vdots \\
\vdots\end{array}$ & $\underline{\hat{\beth}}$ & 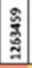 & 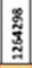 & $\begin{array}{l}\hat{z} \\
\mathbf{z} \\
\end{array}$ & 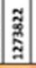 & & 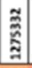 & 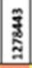 & 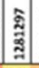 & & & \\
\hline & & & 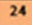 & 24 & 19 & & & & & & & & & & & & & & & & & & & & & & & & & \\
\hline 038 & & $n$ & 24 & 24 & 19 & 24 & 18 & 347 & & & 3 & & & & & 25 & 33 & 26 & & & 30 & 44 & 26 & 22 & 23 & 18 & 38 & 27 & & \\
\hline & & & & & & & & & & $\underline{s}$ & & & & & & & 33 & 26 & & & & & & & & & & & & \\
\hline 8010041 & & 20 & & NA & 19 & 21 & 22 & 249 & & - & z & & & 30 & 30 & 22 & 36 & 26 & 37 & & 30 & 47 & 14 & 18 & 23 & 28 & 38 & 19 & & \\
\hline & & 20 & & 30 & & 21 & & & & 6 & & & & & 30 & 22 & 36 & 26 & & & 30 & & & & & & & & & \\
\hline 501 & & 20 & 20 & 24 & 19 & 15 & 18 & 3 & & 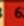 & 22 & & & 30 & 30 & 22 & 36 & 26 & 37 & 17 & 30 & 50 & 26 & 22 & 23 & 18 & 38 & 23 & & \\
\hline & & 20 & 20 & 24 & $=$ & 15 & 18 & & & & 2 & & & 30 & 30 & 22 & 36 & 26 & 37 & 17 & 30 & 50 & 26 & 22 & 23 & 18 & 38 & & & \\
\hline & & $n$ & 24 & 24 & 19 & 24 & 20 & 49 & & 6 & 2 & & & 30 & 30 & 22 & 36 & 26 & 17 & 17 & 30 & 50 & 26 & 22 & 23 & 18 & 38 & 23 & & \\
\hline ESO1 & & 22 & 24 & 24 & 19 & 24 & 20 & 49 & & 6 & 22 & & & 30 & 30 & 22 & 36 & 26 & 37 & 17 & 30 & 50 & 26 & 22 & 23 & 18 & 38 & 23 & & \\
\hline & & 22 & 24 & 24 & 21 & 24 & 20 & 49 & & & 2 & & 26 & 30 & 30 & 22 & 36 & 26 & & 17 & 30 & 50 & 26 & 22 & 23 & 18 & 38 & & & \\
\hline 18000 & & $\mathbf{2 0}$ & 20 & 24 & 19 & 30 & 18 & 347 & 722 & $s$ & 2 & & & 30 & 30 & 22 & 36 & 26 & 37 & 17 & 30 & 50 & 26 & 22 & 23 & 18 & 38 & 23 & & \\
\hline & & 20 & 20 & 24 & & & 18 & 37 & & & & & & 30 & 30 & 22 & 36 & 26 & & & & so & 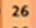 & & & & & & & \\
\hline 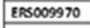 & & 20 & 20 & 24 & 19 & 30 & 18 & 347 & 722 & 5 & 8 & & & 30 & 30 & 22 & 36 & 26 & 37 & 17 & 30 & 50 & 26 & 22 & 23 & 18 & 38 & 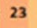 & & \\
\hline & & 20 & 20 & 24 & 19 & 30 & 18 & 47 & & & 8 & & 29 & 30 & & 22 & 36 & 26 & & & 30 & 50 & 2 & 22 & & 18 & 38 & & & \\
\hline ERso1 & & 20 & 20 & 24 & 19 & 30 & 18 & 47 & 7 & 5 & 82 & & 29 & 30 & 30 & 22 & 36 & 26 & 37 & 17 & 30 & so & 26 & 22 & 23 & 18 & 38 & 23 & & \\
\hline & & 20 & & 24 & & & 18 & & & & & & & & 33 & 28 & 33 & & & & 30 & 50 & 26 & $2 \pi$ & & 18 & 38 & & & \\
\hline 100100 & & 18 & 20 & 24 & 19 & 30 & 20 & 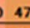 & & $\underline{s}$ & 2 & & 29 & 30 & 30 & 25 & 36 & 26 & 37 & 17 & 30 & 50 & 26 & 22 & 23 & 18 & 38 & - & & \\
\hline & & 20 & 20 & 2 & 19 & 30 & 18 & 349 & & & & & 26 & 24 & 30 & 22 & 36 & 26 & 37 & 17 & 30 & 50 & 26 & 22 & 23 & 18 & 38 & & & \\
\hline & & 20 & 20 & 24 & 19 & 30 & 18 & 3 & 9 & & 2 & & 26 & 24 & 30 & 22 & 36 & 26 & 37 & 17 & 30 & 50 & 26 & 22 & 23 & 18 & 38 & & & \\
\hline & & zo & 20 & 24 & & 30 & 18 & 349 & & & & & & 24 & 30 & 22 & 36 & 26 & 37 & 17 & 30 & 50 & 26 & 22 & 23 & 18 & 38 & & & \\
\hline & & 20 & 20 & 24 & 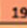 & 30 & 18 & 349 & 9 & & 2 & & 26 & 24 & 30 & 22 & 36 & 26 & 37 & 17 & 30 & 50 & 26 & 22 & 23 & 18 & 38 & & & \\
\hline & & 20 & 20 & 2 & & 524 & 18 & 349 & & & 2 & & & 30 & 30 & 22 & 36 & 26 & 37 & 17 & 30 & 50 & 26 & 22 & 23 & 18 & 38 & & & \\
\hline & & 24 & & 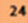 & & & 18 & & & & & & & 30 & 30 & 22 & 5 & 26 & 30 & 17 & 30 & 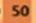 & & 22 & 23 & & & & & \\
\hline & & 20 & 20 & 24 & & 30 & 18 & 3 & 722 & 5 & & & & 30 & 30 & 22 & 36 & 26 & 37 & 17 & 21 & so & 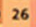 & 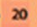 & & 4 & 3 & & & \\
\hline & & 18 & 20 & 24 & & 30 & 18 & 347 & & & & & 29 & 30 & 30 & 25 & 36 & 26 & 41 & 23 & 21 & 32 & 26 & 18 & 23 & & 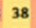 & & & \\
\hline & & 20 & 20 & 24 & 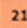 & 15 & 524 & $4 \mathrm{NA}$ & A 22 & & & & 29 & 30 & 33 & 25 & 33 & 26 & 41 & 23 & 21 & 32 & 26 & 18 & 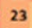 & 28 & 38 & & & \\
\hline & & 20 & & & & & 20 & & & & & & & & & & & 26 & 41 & 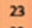 & & & & 18 & & & & & & \\
\hline & & 20 & 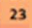 & 24 & & 21 & 18 & 8 & 9 & & & & 29 & 30 & 30 & 22 & 36 & 26 & 41 & 23 & 21 & 32 & 26 & 18 & 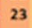 & 28 & 38 & & & \\
\hline & & 18 & & & & & VA & & & & 2 & & 29 & 30 & 33 & 25 & 39 & 28 & 39 & 23 & 21 & 32 & 26 & 18 & 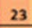 & 28 & 38 & & & \\
\hline & & 20 & 3 & 2 & & & 22 & 2 & 92 & & & & & 30 & 33 & 2 & 33 & 26 & 39 & 23 & 30 & 38 & 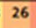 & 18 & 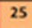 & 26 & 38 & & & \\
\hline & & 20 & & & & & 522 & 24 & & & & & & 0 & 33 & 25 & 33 & 26 & 39 & 23 & 30 & 38 & 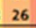 & 18 & & 26 & & & & \\
\hline & & - & 2 & 2 & & 15 & 520 & 0 & & & & & 5 & 30 & 30 & 25 & 36 & 36 & 37 & 20 & 24 & 35 & 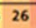 & 20 & 5 & 24 & 32 & & & \\
\hline & & 20 & 20 & 24 & & 15 & 520 & 04 & & & & & & 27 & 30 & 25 & 36 & 36 & 37 & 20 & NA & 35 & 26 & 22 & & & $\mathrm{NA}$ & & & \\
\hline & & 20 & 20 & 24 & & $\sqrt{4}$ & 18 & & & & & & 8 & 27 & 33 & 25 & 36 & $\mathrm{NA}$ & 37 & 23 & 33 & 41 & 2 & 28 & 23 & 120 & 38 & & & \\
\hline & & 20 & 20 & 2 & & 4 & 18 & 8 & & & & & 8 & 27 & 33 & 25 & 36 & 28 & 39 & 23 & 33 & 41 & - & 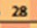 & & 20 & & & & \\
\hline & & 26 & 26 & & & & & & & & & & 26 & 30 & 33 & 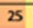 & & 26 & 39 & 3 & 27 & 41 & & 20 & & 26 & & & & \\
\hline & & 20 & 2 & 2 & & 15 & 522 & & & 5 & & & & 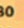 & 27 & 22 & 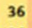 & 3 & 39 & 23 & 24 & 1 & 8 & & & & & & & \\
\hline & & 20 & & & & & & & & & & & & & & & & 2 & & & & & & & & & & & & \\
\hline & & 22 & & & & & & & & & & & & & & & & 22 & & & & & & & & & & & & \\
\hline & & 22 & & & & & & & & & & & & & & & & & & & & 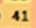 & & & & & & & & \\
\hline & & 22 & & & & & 2 & & & & & & & 30 & 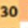 & 25 & 36 & 3 & 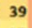 & 23 & 30 & 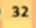 & 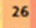 & 1 & 1 & & & & & \\
\hline & & & & & & & & & & & & & & & & & & & & & & & & & & & & & & \\
\hline & & 20 & & & & & & & & & & & & & & & & - & & & & & & & & & & & & \\
\hline & & & & & & & & & & & & & & & & & & & & & & & & & & & & & & \\
\hline & & 20 & & & & & & & & & & & & & 3 & & & & & & & & & & & & & & & \\
\hline & & & & & & & & & & & & & & & & & & & & & & & & & & & & & & \\
\hline & & x & & & & & & & & & & & & & & & & 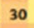 & & & & & & & & & & & & \\
\hline & & & & & & & & & & & & & & & & & & & & & & & & & & & & & & \\
\hline & & 2 & & & & & & & & & & & & & & & & & & & & & & & & & & & & \\
\hline & & & & & & & & & & & & & & & & & & & & & & & & & & & & & & \\
\hline & & 26 & & & & & & & & & & & & & & & & & & & & & & & & & & & & \\
\hline & & & & & & & & & & & & & & & & & & & & & & & & & & & & & & \\
\hline & & & & & & & & & & & & & & & & & & & & & & & & & & & & & & \\
\hline & & & & & & & & & & & & & & & & & & & & & & & & & & & & & & \\
\hline
\end{tabular}

Fig. 1 Microsatellite linkage disequilibrium and haplotypes across chromosome 6 of Plasmodium falciparum from the Gambia in 2008. a Mean pairwise linkage disequilibrium $\left(r^{2}\right)$ plotted against the physical position (base pairs on chromosome) of 775 polymorphic repeat loci across chromosome 6. Microsatellite genotypes were derived from short sequence reads of 54 P. falciparum isolates collected in 2008. The shaded box delineates the region of peak LD, representing the chromosome 6 selective sweep. $\mathbf{b}$ Zoom-in on $174 \mathrm{~kb}$ region of peak LD on panel 'a', showing the haplotypes from alleles (repeat-number) of 28 polymorphic microsatellite loci with at least three alleles. The first column in panel 'b' is the European Nucleotide Archive sample/data ID and the following columns are physical positions (bp) of chosen repeat loci on the chromosome. The numbers in cells are the repeat lengths and colour intensity for each cell in a column increases with increase in repeat lengths. Haplotypes defined by matching of loci alleles across the region are shown as grouped cells shaded with alternative intensities of grey and named arbitrarily from $\mathrm{A}$ to $\mathrm{H}$ 
within a window of $72.7 \mathrm{~kb}(1,201,161-1,273,822 \mathrm{bp})$ with the most intact haplotypes, 8 major long-range haplotypes at frequencies between 3.7 and $16.7 \%$ were identified. The rest of the isolates had partial and recombinant forms of this haplotype.

\section{Analysis of microsatellite loci in temporal populations from The Gambia}

Laboratory genotyping was performed on 13 loci spanning $179.5 \mathrm{~kb}$ of the selective sweep region for 439 isolates from population samples taken in $1984(\mathrm{~N}=56)$, $1991(\mathrm{~N}=67), 2008(\mathrm{~N}=166)$ and 2014 ( $\mathrm{N}=150)$. These loci were separated by a mean distance of $15 \mathrm{~kb}$ $(3.2-20.7 \mathrm{~kb})$. The mean virtual heterozygosity across all loci was 0.77 , ranging from 0.47 to 0.92 (highest in the dinucleotide repeat loci and decreasing with increasing repeat unit size). Pairwise linkage disequilibrium between adjacent loci revealed two peaks with $r^{2}$ values of 0.06 and 0.12 respectively for loci SSR13 against SSR15 separated by $16 \mathrm{~kb}(1,135,849-1,151,868 \mathrm{bp})$ and for loci SSR21 against SSR23 separated by $20.5 \mathrm{~kb}(1,207,751-$ 1,228,301 bp), (Fig. 2a). Per locus pairwise population Fst values were generally low (0-0.25). Higher indices were observed when comparing 1984 and 1991 to more recent populations from 2008 and 2014 for SSR3 (1,078,732 bp), SSR21 (1,207,751 bp) and SSR25 (1,249,025 bp), (Fig. 2b). Values between 1894 and 1991 were low for all loci except for SSR3. The lowest level of differentiation was between 2008 and 2014 populations, with nine out of 13 loci showing non-significant Fst values (Additional file 1: Tables-sheet: Fst-13Loci). Following Bayesian simulation of pairwise population Fst distribution at each locus, seven (SSR1, SSR3, SSR5, SSR7, SSR11, SSR13 and SSR19) out of the selected 13 loci had a pattern of variation between populations that deviated from neutral expectations (Additional file 4a). Of these, SSR13 (Pf3D7_06_v3: 1135849) had the strongest signature of deviation from neutrality. This locus is located within the Pf3D7_0628100 sequence, which codes for 6-pyruvoyltetrahydropterin synthase (PTPS). The other six loci had lower Fst and high q-values. Three loci: SSR19 (Pf3D7_06_v3:1188628), SSR21 (Pf3D7_06_v3:1207757) and SSR23 (Pf3D7_06_v3:1228301), also showed significant deviation of Fst and heterozygosity than expected under neutrality (Additional file 4b). These loci span the region of peak LD detected (Fig. 2a). There are 11 genes encoded in the region with highest LD and Fsts, which includes 3 transferases, a transportin, a transporter, a synthetase, and a synthase (Fig. 2c; Additional file 1: Tables-sheet: Genes_in_Sweep). The entire $179.5 \mathrm{~kb}$ region analysed with 13 microsatellite loci across temporal populations, codes for 36 genes, which are involve in a variety of metabolic processes (Additional file 1: Tablessheet: Genes_in_Sweep).

\section{Shared long-range haplotypes}

With complete matching at all 13 loci, 243 (55.4\%) isolates had unique haplotypes while 196 were shared by at least 2 isolates from the four temporal populations. Of the latter, 18 isolates shared the longest uninterrupted haplotype only from the 1991, 2008 and 2014 populations (Fig. 3). Recombinant and partial forms of this major haplotype were found in 39 other isolates from the same populations. The structure of this long-range haplotype was most conserved at five contiguous loci (SSR13SSR21) spanning 71.9 kb (1,135,849-1,207,757 bp), (Fig. 3). With complete matching at all five loci within this conserved window, 4 (5.9\%), 28 (16.9\%) and 25 (16.6\%) isolates from 1991, 2008 and 2014 respectively had this haplotype group. This represents $13 \%$ of all isolates from these populations (Fig. 3). There were 40 other haplotypes shared by $2-13$ isolates from the four populations. Three of these haplotypes were present in the 1984 population of which 2 were seen in single isolates from 1991 and 2008 (Table 1). Nine haplotypes from 1991 were present at higher frequencies in 2008 and 2014.

The frequency of shared haplotypes was higher in later years; 8.9, 19.4, 60.8 and $51.3 \%$ for 1984, 1991, 2008 and 2014 respectively. Using loci with peak LD as focus (SSR13 and SSR21), two long-range LD tracks of $\sim 100 \mathrm{~kb}$ each could be distinguished (Additional file 5). The block determined by SSR13 was present in the earliest population sample from 1984 (Additional file 6). The types and the frequencies of these haplotypes increased in the more recent populations from 2008 and 2014. The haplotypes defined by SSR21 were dominant from 2008 and maintained a similar structure in 2014 .

\section{Discussion}

This is the first study describing extended haplotypes of microsatellite loci within a chromosome 6 region of $P$. falciparum previously determined with SNPs to be under selection. The microsatellite alleles derived from Illumina short read sequence data of clinical isolates collected in 2008 detected a $200 \mathrm{~kb}$ window of significantly elevated linkage disequilibrium that overlaps with the previously determined selective signature on the chromosome $[6,7,16]$. It shows microsatellites and linkage disequilibrium to be sensitive in detecting regions under selection as reported previously $[14,17]$. The use of microsatellites as markers has the potential to focus on very recent events. In contrast to SNPs, Higher mutation rates will also provide sufficient variation in closely related populations, and would reveal only recent selective sweeps. As our focus here was over a 


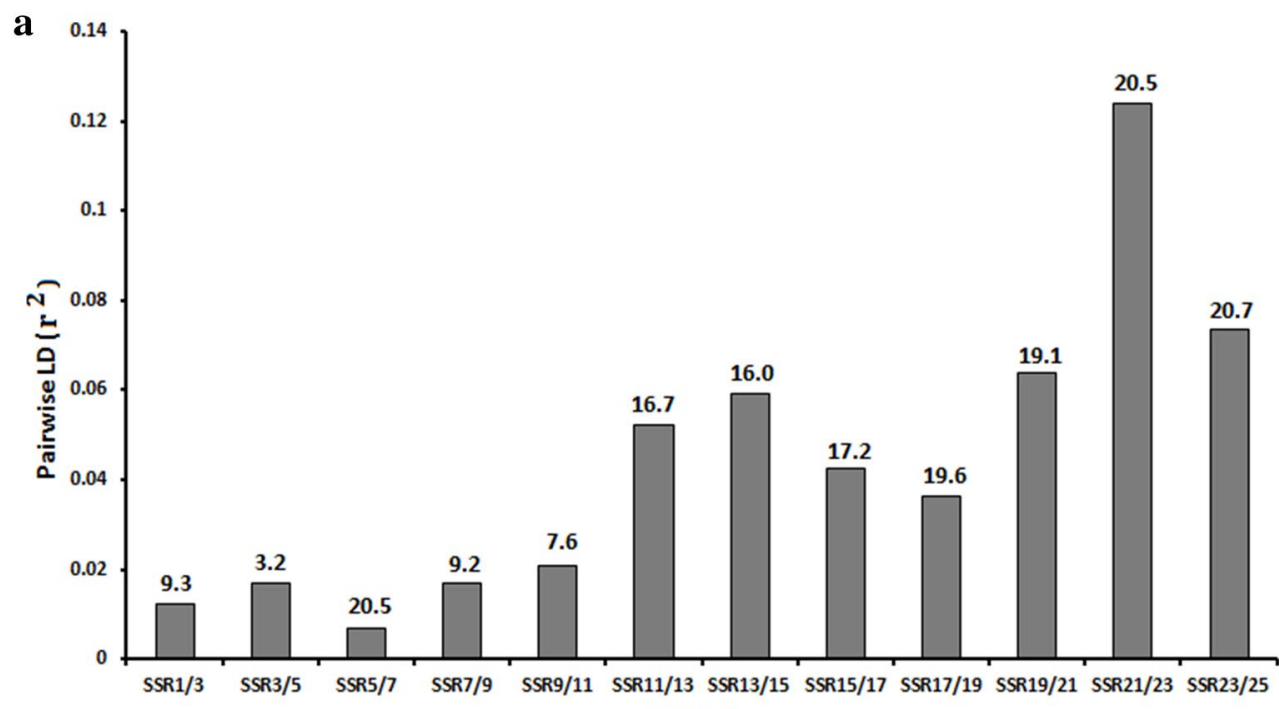

b Loci Pairs

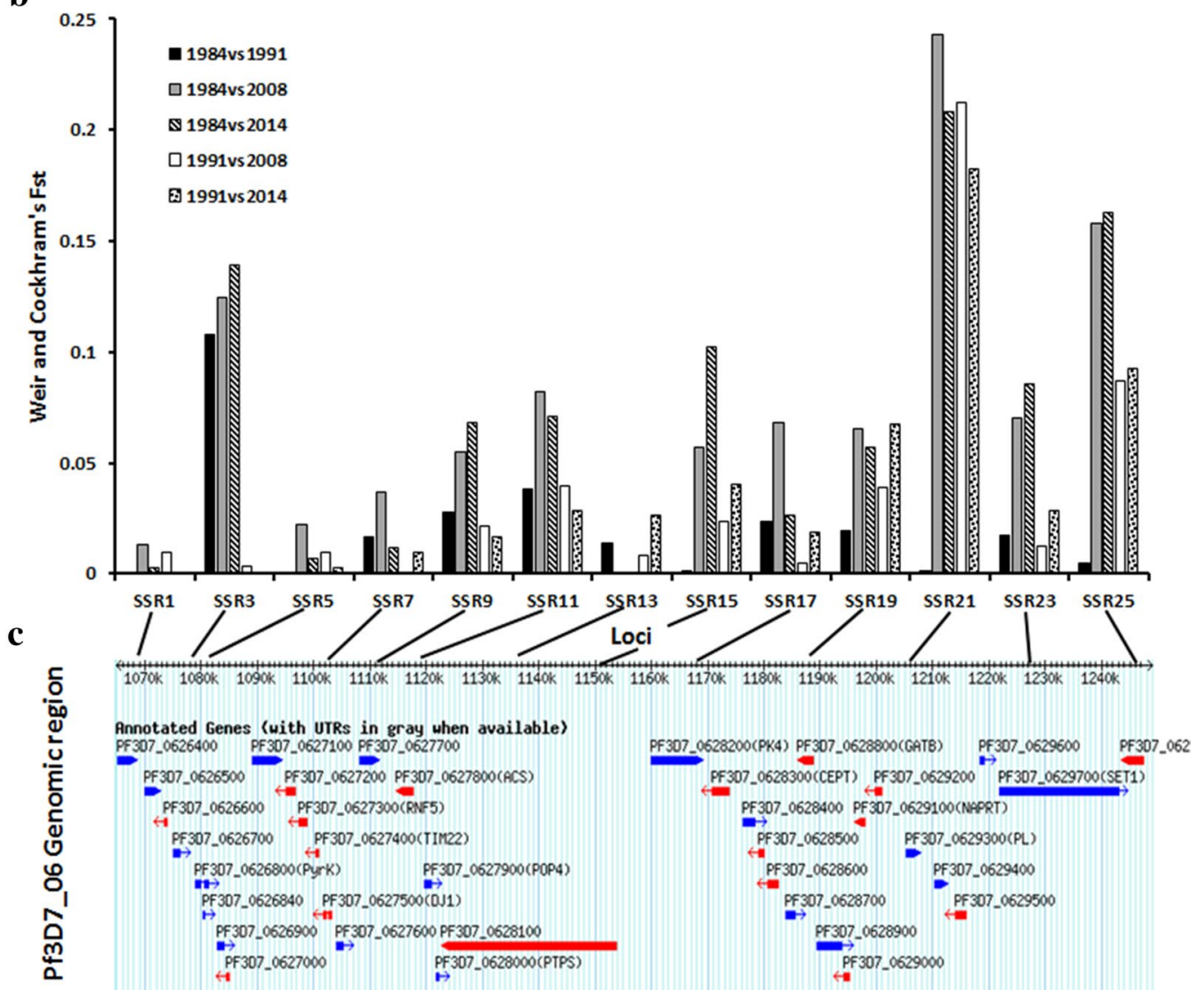

Fig. 2 Analysis of genotypes at 13 microsatellite loci chosen across the $179.5 \mathrm{~kb}$ signature of selection on chromosome 6 of $P$. falciparum. a Extent of pairwise linkage disequilibrium $\left(r^{2}\right)$ between adjacent loci as columns labelled on the $x$-axis with the arbitrary names of the loci pair. The physical distance $(\mathrm{kb})$ separating the pair of loci for which the $\mathrm{r}^{2}$ was determined is shown on top of each column. $\mathbf{b}$ Weir and Cockeram's Fst at each locus for pairs of temporal populations compared. Each column represents the extent of Fst between population pairs. c Snapshot of genomeview display in PlasmoDB for the region covered by the 13 loci (http://www.plasmoDB.org). The connecting lines from locus names in panel ' $\mathbf{b}^{\prime} \mathbf{x}$-axis maps the physical positions (kb) of each locus on the genome ruler in panel ' $\mathbf{c}$ '. The blue and red bars in panel ' $\mathbf{c}$ ' represent coding sequences with the arrow head showing the direction of translation. The gene ID of each coding sequence is shown above each bar 


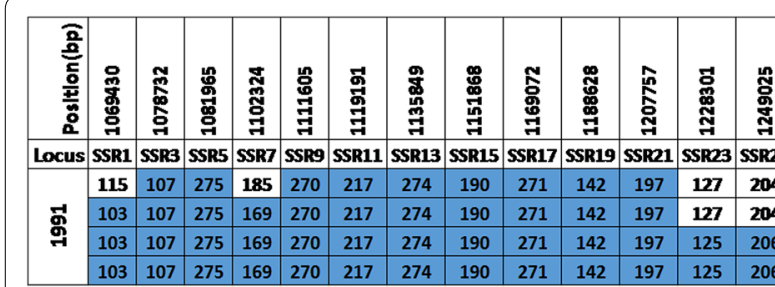

\begin{tabular}{|c|c|c|c|c|c|c|c|c|c|c|c|c|c|}
\hline \multirow{28}{*}{ : } & 111 & 135 & 283 & 173 & 246 & 217 & 274 & 190 & 271 & 142 & 197 & 125 & 206 \\
\hline & 111 & 135 & 283 & 173 & 246 & 217 & 274 & 190 & 271 & 142 & 197 & 125 & 206 \\
\hline & 103 & 107 & 275 & 169 & 268 & 217 & 274 & 190 & 271 & 142 & 197 & 127 & 204 \\
\hline & 115 & 107 & 275 & 169 & 250 & 217 & 274 & 190 & 271 & 142 & 197 & 127 & 204 \\
\hline & 103 & 107 & 275 & 169 & 252 & 217 & 274 & 190 & 271 & 142 & 197 & 125 & 204 \\
\hline & 103 & 107 & 275 & 169 & 282 & 217 & 274 & 190 & 271 & 142 & 197 & 125 & 206 \\
\hline & 103 & 107 & 275 & 169 & 270 & 207 & 274 & 190 & 271 & 142 & 197 & 125 & 206 \\
\hline & 103 & 107 & 275 & 169 & 270 & 215 & 274 & 190 & 271 & 142 & 197 & 125 & 206 \\
\hline & 115 & 107 & 275 & 169 & 270 & 217 & 274 & 190 & 271 & 142 & 197 & 125 & 206 \\
\hline & 101 & 107 & 275 & 169 & 270 & 217 & 274 & 190 & 271 & 142 & 197 & 125 & 196 \\
\hline & 103 & 107 & 275 & 169 & 270 & 217 & 274 & 190 & 271 & 142 & 197 & 127 & 206 \\
\hline & 103 & 107 & 275 & 169 & 270 & 217 & 274 & 190 & 271 & 142 & 197 & 125 & 204 \\
\hline & 103 & 107 & 275 & 169 & 270 & 217 & 274 & 190 & 271 & 142 & 197 & 125 & 206 \\
\hline & 103 & 107 & 275 & 169 & 270 & 217 & 274 & 190 & 271 & 142 & 197 & 125 & 206 \\
\hline & 103 & 107 & 275 & 169 & 270 & 217 & 274 & 190 & 271 & 142 & 197 & 125 & 206 \\
\hline & 103 & 107 & 275 & 169 & 270 & 217 & 274 & 190 & 271 & 142 & 197 & 125 & 206 \\
\hline & 103 & 107 & 275 & 169 & 270 & 217 & 274 & 190 & 271 & 142 & 197 & 125 & 206 \\
\hline & 103 & 107 & 275 & 169 & 270 & 217 & 274 & 190 & 271 & 142 & 197 & 125 & 206 \\
\hline & 103 & 107 & 275 & 169 & 270 & 217 & 274 & 190 & 271 & 142 & 197 & 125 & 206 \\
\hline & 103 & 107 & 275 & 169 & 270 & 217 & 274 & 190 & 271 & 142 & 197 & 125 & 206 \\
\hline & 103 & 109 & 275 & 169 & 270 & 217 & 274 & 190 & 271 & 142 & 197 & 125 & 206 \\
\hline & 103 & 113 & 275 & 169 & 270 & 217 & 274 & 190 & 271 & 142 & 197 & 125 & 206 \\
\hline & 103 & 133 & 275 & 169 & 270 & 217 & 274 & 190 & 271 & 142 & 197 & 125 & 206 \\
\hline & 105 & 111 & 299 & 175 & 270 & 217 & 274 & 190 & 271 & 142 & 197 & 125 & 206 \\
\hline & 111 & 107 & 277 & 183 & 270 & 217 & 274 & 190 & 271 & 142 & 197 & 125 & 206 \\
\hline & 111 & 123 & 267 & 169 & 270 & 217 & 274 & 190 & 271 & 142 & 197 & 123 & 208 \\
\hline & 117 & 129 & 287 & 163 & 270 & 229 & 274 & 190 & 271 & 142 & 197 & 127 & 204 \\
\hline & 99 & 111 & 271 & 175 & 264 & 227 & 274 & 190 & 271 & 142 & 197 & 125 & 204 \\
\hline \multirow{26}{*}{ 월 } & & & & & & & & & & & & & \\
\hline & 109 & 107 & 277 & 169 & 252 & 217 & 274 & 190 & 271 & 142 & 197 & 125 & 190 \\
\hline & 111 & 107 & 275 & 169 & 270 & 197 & 274 & 190 & 271 & 142 & 197 & 125 & 202 \\
\hline & 107 & 107 & 275 & 169 & 270 & 217 & 274 & 190 & 271 & 142 & 197 & 125 & 196 \\
\hline & 101 & 97 & 307 & 169 & 270 & 217 & 274 & 190 & 271 & 142 & 197 & 121 & 202 \\
\hline & 103 & 127 & 287 & 169 & 270 & 217 & 274 & 190 & 271 & 142 & 197 & 127 & 204 \\
\hline & 103 & 127 & 287 & 169 & 270 & 217 & 274 & 190 & 271 & 142 & 197 & 127 & 204 \\
\hline & 103 & 127 & 287 & 175 & 246 & 217 & 274 & 190 & 271 & 142 & 197 & 125 & 206 \\
\hline & 103 & 127 & 287 & 175 & 246 & 217 & 274 & 190 & 271 & 142 & 197 & 125 & 206 \\
\hline & 103 & 107 & 275 & 149 & 270 & 217 & 274 & 190 & 271 & 142 & 197 & 125 & 206 \\
\hline & 103 & 107 & 275 & 169 & 270 & 217 & 274 & 190 & 271 & 142 & 197 & 125 & 206 \\
\hline & 103 & 107 & 275 & 169 & 270 & 217 & 274 & 190 & 271 & 142 & 197 & 125 & 206 \\
\hline & 117 & 107 & 275 & 169 & 270 & 217 & 274 & 190 & 271 & 142 & 197 & 125 & 206 \\
\hline & 103 & 107 & 275 & 189 & 270 & 217 & 274 & 190 & 271 & 142 & 197 & 125 & 206 \\
\hline & 107 & 107 & 277 & 169 & 270 & 217 & 274 & 190 & 271 & 142 & 197 & 125 & 206 \\
\hline & 107 & 107 & 279 & 169 & 270 & 217 & 274 & 190 & 271 & 142 & 197 & 125 & 206 \\
\hline & 107 & 107 & 299 & 169 & 270 & 217 & 274 & 190 & 271 & 142 & 197 & 125 & 206 \\
\hline & 107 & 141 & 283 & 169 & 270 & 217 & 274 & 190 & 271 & 142 & 197 & 125 & 206 \\
\hline & 117 & 127 & 273 & 177 & 270 & 217 & 274 & 190 & 271 & 142 & 197 & 125 & 206 \\
\hline & 107 & 141 & 303 & 179 & 270 & 217 & 274 & 190 & 271 & 142 & 197 & 125 & 206 \\
\hline & 101 & 101 & $\mathbf{2 8 1}$ & 163 & 270 & 229 & 274 & 190 & 271 & 142 & 197 & 125 & 206 \\
\hline & 117 & 129 & 287 & 163 & 270 & 229 & 274 & 190 & 271 & 142 & 197 & 125 & 206 \\
\hline & 117 & 129 & 287 & 163 & 270 & 229 & 274 & 190 & 271 & 142 & 197 & 125 & 206 \\
\hline & 91 & 121 & 269 & 203 & 254 & 227 & 274 & 190 & 271 & 142 & 197 & 125 & 206 \\
\hline & 99 & 111 & 289 & 171 & 256 & 217 & 274 & 190 & 271 & 142 & 197 & 121 & 206 \\
\hline & 91 & 125 & 287 & 175 & 252 & 27 & 274 & 190 & 271 & 142 & 197 & 125 & 204 \\
\hline
\end{tabular}

relatively short period (23 years), any deviations in variability in the linked loci will be marking selection closer to the target locus. Several long-range haplotypes were detected in the sweep region suggesting more than a single founder haplotype under selection. To validate and examine
Fig. 3 Structure of the dominant haplotype derived from fragment analysis of 13 microsatellite loci spanning $179.5 \mathrm{~kb}$ of chromosome 6 selective signature in P. falciparum. The first row shows the physical positions (bp) of each locus and the arbitrary names of the loci are presented in each column of the second row. The years of sampling for each population is shown in merged rows of the first column while the following columns are microsatellite loci positions on the chromosome. For each population, the rows present the haplotype of a sample across the 13 loci. The numbers in each cell is the allele (fragment size in bp determined from capillary electrophoresis) for a sample at each locus. Alleles of the major haplotype are shaded in blue

haplotype structure and frequencies in this genomic region, four populations of $P$. falciparum isolates from The Gambia spanning 30 years (1984-2014) were characterized with a subset of 13 loci across $179.5 \mathrm{~kb}$. During the 30 years, the first line chemotherapy for malaria changed from chloroquine to SP (2002) and then ACT (2008). These loci were highly diverse in all temporal $P$. falciparum populations genotyped. Similar to genomic loci from Illumina short reads, we detected strong linkage disequilibrium and several extended haplotypes. Sweeps lead to increase in LD when they are still in progress [18]. Thus, selection could have started as early as 1984 and continued into recent populations. This is also supported by increase in the frequencies of long-range haplotypes in the region from 1984 to recent populations. However, for the most common combination of multilocus alleles (from 1,135,849$1,207,757 \mathrm{bp}$ ), only two haplotypes found in the earliest population (1984) were present in single isolates from 1991 and 2008. On the other hand, other haplotypes upstream of this region were already at high frequencies in 1984. This would suggest selection on more than one locus or more than one event occurring prior to the 80s and between $80 \mathrm{~s}$ and 90s. Antifolate anti-malarials were not officially used earlier than the year 2002 in the Gambia and so selection around these earlier years cannot be due to SP. The results here are against suggestions that anti-malarial SP might be the driver of selection in this region [5].

Finding a variety of long-range haplotypes at low to moderate frequencies in all temporal populations is contrary to the classical outcome of hard selective sweeps, where one haplotype carrying the favourable mutation rises in frequency to fixation [19]. With 36 genes coded for by the region with the selective signature, selection could be polygenic or both diversifying and directional selection may be acting on different loci in the region. While identifying the exact loci being selected remain beyond this current analysis, three genes within the region might be under non-neutral variation from previous SNP analysis and the analysis herein. The first gene is the acetyl-CoA synthetase (PF3D7_0627800) which was predicted as being under balancing selection [16]. Balancing selection increases diversity around a selected 
Table 1 Frequencies of core haplotypes spanning $71.5 \mathrm{~kb}$ across the selective sweep region on chromosome 6 of $P$. falciparum from the Gambia collected in 1984, 1991, 2008 and 2014

\begin{tabular}{|c|c|c|c|c|c|}
\hline Haplotype & 1984 & 1991 & 2008 & 2014 & Frequency \\
\hline 274190271142197 & & 4 & 28 & 25 & 13.0 \\
\hline 280181277144197 & & & 7 & 6 & 3.0 \\
\hline 274190271144197 & & 1 & 7 & 1 & 2.1 \\
\hline 274190275144197 & & & & 8 & 1.8 \\
\hline 280181279142197 & & & 6 & 1 & 1.6 \\
\hline 274181269142197 & & 1 & 5 & & 1.4 \\
\hline 274181271142197 & & 1 & 3 & 2 & 1.4 \\
\hline 274181281138225 & & & 3 & 2 & 1.1 \\
\hline 274184285126197 & & 1 & 2 & 2 & 1.1 \\
\hline 274181269140197 & & & 4 & & 0.9 \\
\hline 274181285138197 & & 1 & & 3 & 0.9 \\
\hline 280181279144197 & & & 1 & 3 & 0.9 \\
\hline 280184271142197 & & & 4 & & 0.9 \\
\hline 268178271142197 & & & 1 & 2 & 0.7 \\
\hline 274178275144197 & & & & 3 & 0.7 \\
\hline 274178279128213 & & 1 & 2 & & 0.7 \\
\hline 274181273136197 & 2 & & 1 & & 0.7 \\
\hline 274181279142197 & & & 3 & & 0.7 \\
\hline 274190275144205 & & & & 3 & 0.7 \\
\hline 280184285142197 & & & 2 & 1 & 0.7 \\
\hline 298178275144197 & & & & 3 & 0.7 \\
\hline 268181265144197 & & & 2 & & 0.5 \\
\hline 274172271142197 & & & & 2 & 0.5 \\
\hline 274175271150197 & 1 & 1 & & & 0.5 \\
\hline 274178277144221 & & & 2 & & 0.5 \\
\hline 274181269144197 & & & 2 & & 0.5 \\
\hline 274181271128201 & 2 & & & & 0.5 \\
\hline 274181271138197 & & & 2 & & 0.5 \\
\hline 274181271146197 & & 1 & 1 & & 0.5 \\
\hline 274181275150197 & & & 2 & & 0.5 \\
\hline 274181275154213 & & & & 2 & 0.5 \\
\hline 274181281138197 & & & 2 & & 0.5 \\
\hline 274181291132225 & & & 2 & & 0.5 \\
\hline 274184283126197 & & & 1 & 1 & 0.5 \\
\hline 274184283138229 & & & 2 & & 0.5 \\
\hline 274190269142197 & & & 2 & & 0.5 \\
\hline 274190271142217 & & & & 2 & 0.5 \\
\hline 274190277144197 & & & 1 & 1 & 0.5 \\
\hline 280181271142197 & & 1 & & 1 & 0.5 \\
\hline 280181277142197 & & & & 2 & 0.5 \\
\hline 280181281142197 & & & 1 & 1 & 0.5 \\
\hline
\end{tabular}

The first column shows the haplotypes defined by a contiguous string of PCR fragment sizes in base pairs for five neighbouring loci within this region. The following columns show the number of isolates from each year with the haplotype. The last column presents the frequencies for the entire population locus and would drive the multiplicity of haplotypes. Long-term maintenance of such polymorphisms and reduction in recombination would increase LD in neutral neighbouring loci around the focus of selection. The second gene in the region is 6-pyruvoyltetrahydropterin synthase, PTPS (PF3D7_0628000) which has a direct role in pterin metabolism and folate salvage pathways in $P$. falciparum [20]. Folate salvage is a major mechanism of SP resistance. The increase in complete long-range haplotype frequencies in the 2008 population provides support to selection from sulfonamides. However, haplotypes around the PTPS gene that is involved in sulfonamide metabolic processes seemed to have emerged earlier than 2002. Since SP was not in use when the earlier populations here were collected, selection of such pathways might be as a result of non-antimalarial sulfonamides, such as sulfamethoxazole/trimethoprim (cotrimoxazole), that have been used across Africa for a long time. Thirdly there is a phospholipase A2, PF3D7_0629300 (phosphatidylcholine-sterol acyltransferase, putative gene), which by Bayesian analysis of Fst distribution of ssr loci, may be under selection. The repeat locus within this gene showed the highest Fst and LD. Phospholipase activity increases membrane permeability and stimulation of many enzymes associated with processes that disrupt cytoskeleton and membrane structure [21]. They are also strongly inhibited by quinoline anti-malarials [22, 23]. Hence, the lipase may also be under directional selection from quinolines used in the 1990s. Chloroquine in particular was the most widely used quinolines against malaria before the $90 \mathrm{~s}$ and could have been driving selection. Antifolate SP replaced chlorquine from 2002 to 2008.

There was increase in frequencies of partial forms of haplotypes in the 2014 population, 6 and 12 years after the withdrawal of SP and chloroquine respectively as first line treatment. However, SP is still in use for intermittent preventive treatment and is now a component of seasonal malaria chemoprophylaxis [24]. The non-antimalarials antibiotics cotrimoxazole and derived quinolines were introduced in clinical practice in the 1960s while most quinoline antibiotics came into use in the 1980s. Cotrimoxazole in particular has been shown to be efficacious against malaria and thus might have been a source of selection on folate metabolism pathways for over half a century. If selection is acting on the phospholipase, due to quinolones, this could also explain the early origin of long-range haplotypes. Moreover, anti-malarial quinolines (e.g., quinine) have been in use against malaria for over is 400 years [25]. With SP being a component of SMC, increased frequencies of the sweep haplotypes in future analysis of 
SMC treated populations could confirm its role in selection. Meanwhile, analysis of populations with the earliest records of quinolone resistance such as Latin America could help resolve the involvement of quinolones as a selective force on this genomic region. With increasing accumulation of genomic data, this will be possible in the near future.

\section{Conclusions}

This study has observed multiple long-range haplotypes of a region on P. falciparum chromosome 6 maintained over more than two decades in The Gambia. The identity of the target of selection remains unknown as the narrowest region implicated contains 24 genes involved in various metabolic processes. It is unlikely to be a result of anti-malarial drug selection only, as extended haplotypes existed before the widespread use of antifolates for malaria treatment, and before chloroquine resistance alleles had become locally common. It is possible that off-target drug action from antibiotics may have been responsible, or some completely different process may be maintaining extended haplotypes in this region of the parasite genome.

\section{Additional files}

Additional file 1. Additional tables of raw illumina derived genotypes, repeat motif distribution, microsatellite fragment sizes and analysis, PCR primers and probes.

Additional file 2. Correlation of repeat length polymorphisms determined from Illumina short sequence reads and PCR fragment size analyses for three microsatellite loci (SSR1, SSR9 and SSR15) on chromosome 6 of $P$. falciparum. Each point is the Illumina repeat length ( $y$-axis) plotted against the PCR fragment size ( $x$-axis) in base pairs for a sample. The trend in correlation is shown as a broken blue line.

Additional file 3. Mean haplotype frequencies for microsatellite loci within windows of $10 \mathrm{~kb}$ across chromosome 6 . The smoothed line is derived from haplotype frequency averages between adjacent windows plotted against the physical position on the chromosome. A total of 775 microsatellite loci were analysed. There were elevated haplotype frequencies spanning a $100 \mathrm{~kb}$ region that overlaps with the previously determined chromosome selective signature.

Additional file 4. Bayesian analysis of Fst distribution for 13 chromosome 6 microsatellites genotyped in 4 temporal populations in The Gambia. (a) Simulated WC -Fst is plotted against q-values following 10,000 iterations in Bayescan. Seven out of 13 loci (unshaded points) are under non-neutral variation at $5 \%$ false discovery rate. The locus with the highest positive deviation is at position $1207 \mathrm{~kb}$ in the phospholipase gene. (b) Distribution of Fst against a derivative of heterozygosity. Loci beyond the $95 \%$ confidence interval for the distribution of Fst and heterozygosity are candidate targets of possible selection.

Additional file 5. Linkage disequilibrium map for 13 microsatellite loci across $179.5 \mathrm{~kb}$ of $P$. falciparum chromosome 6. Each plot presents data from a single population labelled at the top left of the plot panel with the year the isolates were collected. Loci are labelled at the top of each plot. Boxes in each plot represents LD between loci in blue or red indicating non-significant or highly significant $r^{2}$ values between alleles of the loci. More intense colours represent extreme values of significance.
Additional file 6. Haplotype homozygosity across $179.5 \mathrm{~kb}$ region of chromosome 6 determined from 13 microsatellite loci by fragment analysis of temporal populations from the Gambia. Each plot shows bifurcation of haplotypes from the core locus with the thickness of the blue shaded lines corresponding to the frequency of the long-range haplotype. The horizontal axis shows the physical positions on the chromosome while the broken vertical line marks the position of the focal locus from which extended haplotypes were defined to the left and right. Plots on the left column were derived from haplotype structures at SSR13 (1,135,849 bp) for each population from 1984, 1991, 2008 and 2014 in rows from top to bottom and labelled on the right. The right column of plots present extended haplotypes derived from SSR21 (1,207,757 bp) for same populations. SSR13 and SSR21 showed the lowest and highest Fst values respectively within the region of elevated linkage disequilibrium.

\section{Authors' contributions}

AAN designed studies, protocols and assays. He wrote the manuscript. BD performed laboratory microsatellite genotyping. AW assisted in genomic microsatellite screening from Illumina data. SC participated in sample collection, DNA extraction and microsatellite genotyping. ND contributed to oversight of laboratory processes and commented on the manuscript. DJ supported with statistical analyses. UDA advised on sampling and commented on the manuscript. DC advised on the study design, data analysis, and development of the manuscript. All authors read and approved the final manuscript.

\section{Author details}

${ }^{1}$ Medical Research Council, Gambia Unit, Atlantic Road, Fajara, P.O. Box 273, Banjul, The Gambia. ${ }^{2}$ London School of Hygiene and Tropical Medicine, Keppel Street, London, UK

\section{Acknowledgements}

We thank Drs. Samuel Assefa and Craig Duffy for their technical assistance and the helpful discussions in data generation and analysis. We are grateful to Drs Chris J. Drakeley, Colin J. Sutherland and Muminatou Jallow for the archival samples accessed via the MRC Gambia biobank. This work was supported by funds from an MRC Career Development fellowship to Dr. Alfred Amambua Ngwa through the WT/MRC/DFID/EDCTP Global Health Scheme.

\section{Competing interests}

All authors declare that they have no competing interests.

\section{Availability of data and material}

Short sequence reads; the study makes use of short sequence reads from next generation sequencing by Illumina technologies. All reads used from microsatellite genotyping are available via the European Nucleotide Archive (ENA) The file names have been included in the Additional file 1-RepeatSeq calls.

All other data related to the manuscript are present in Additional file 1 as separate Excel worksheets. This includes:

1. Filtered-Pf06Sweep alleles from Genotan: Alleles filtered for chromosome 6 by missingness

2. Allele_Types: Analysis of length and motif distribution for microsatellite alleles from NGS.

3. Diploidised_fragment_sizes: Fragment sizes of PCR products from 13 selected loci.

4. Fst_13Loci:WC Fst for all pairs of populations per loci.

5. Targeted_13_ssr_stats_primers: Primers and probes used in genotyping.

6. Poppr_MLGs: Multilocus genotypes by Poppr package.

7. PopGenstats: Population genetics statistics for insilico derived microsatellite genotypes.

8. LD_and_HaplotypeFrequencies: LD and allele frequencies for NGS microsatellite genotypes.

9. Genes_in_Sweep: Gene within the chromosome 6 sweep spanning $1,100,277-1,242,922 \mathrm{~kb}$.

10.Illumina_vs_FragmentAnalysis: Correlation of Illumina and fragment analysis allele sizes for ssr loci 1, 9 and 15 . 


\section{Ethics approval and consent to participate}

The study reported here was reviewed and approved by the joint Gambian Government/MRC Ethical Committee. This study used archived material collected and preserved for parasitological research following direct consent or assent from adults or minors respectively from the study populations. The data has no direct link to patient information and only parasite material and genetic analysis was performed.

\section{Funding}

The work in this manuscript was principally funded from a Career Development Award by the Medical Research Council, UK, to Dr. Alfred Amambua Ngwa under the MRC/DFID/EDCTP scheme. Short Read sequences from Next Generation Sequencing were acquired in collaboration with the Centre for Global Health Genomics funded by MRC, UK.

Received: 5 June 2016 Accepted: 6 October 2016

Published online: 21 October 2016

\section{References}

1. Mu J, Myers RA, Jiang H, Liu S, Ricklefs S, Waisberg M, et al. Plasmodium falciparum genome-wide scans for positive selection, recombination hot spots and resistance to antimalarial drugs. Nat Genet. 2010;42:268-71.

2. Volkman SK, Sabeti PC, DeCaprio D, Neafsey DE, Schaffner SF, Milner DA $\mathrm{Jr}$, et al. A genome-wide map of diversity in Plasmodium falciparum. Nat Genet. 2007;39:113-9.

3. Mu J, Awadalla P, Duan J, McGee KM, Keebler J, Seydel K, et al. Genomewide variation and identification of vaccine targets in the Plasmodium falciparum genome. Nat Genet. 2007;39:126-30.

4. Nwakanma DC, Duffy CW, Amambua-Ngwa A, Oriero EC, Bojang KA, Pinder $M$, et al. Changes in malaria parasite drug resistance in an endemic population over a 25-year period with resulting genomic evidence of selection. J Infect Dis. 2014;209:1126-35.

5. Park DJ, Lukens AK, Neafsey DE, Schaffner SF, Chang H-H, Valim C, et al. Sequence-based association and selection scans identify drug resistance loci in the Plasmodium falciparum malaria parasite. Proc Natl Acad Sci USA. 2012;109:13052-7.

6. Amambua-Ngwa A, Park DJ, Volkman SK, Barnes KG, Bei AK, Lukens AK, et al. SNP genotyping identifies new signatures of selection in a deep sample of west African Plasmodium falciparum malaria parasites. Mol Biol Evol. 2012;29:3249-53.

7. Mobegi VA, Duffy CW, Amambua-Ngwa A, Loua KM, Laman E, Nwakanma $D C$, et al. Genome-wide analysis of selection on the malaria parasite Plasmodium falciparum in West African populations of differing infection endemicity. Mol Biol Evol. 2014;31:1490-9.

8. Borrmann S, Straimer J, Mwai L, Abdi A, Rippert A, Okombo J, et al. Genome-wide screen identifies new candidate genes associated with artemisinin susceptibility in Plasmodium falciparum in Kenya. Sci Rep. 2013;3:3318.

9. Ocholla H, Preston MD, Mipando M, Jensen ATR, Campino S, MacInnis B, et al. Whole-genome scans provide evidence of adaptive evolution in Malawian Plasmodium falciparum isolates. J Infect Dis. 2014;210:1991-2000.

10. Samad H, Coll F, Preston MD, Ocholla H, Fairhurst RM, ClarkTG. Imputation-based population genetics analysis of Plasmodium falciparum malaria parasites. PLoS Genet. 2015;11:e1005131.

11. Wendler JP, Okombo J, Amato R, Miotto O, Kiara SM, Mwai L, et al. A genome wide association study of Plasmodium falciparum susceptibility to 22 antimalarial drugs in Kenya. PLoS ONE. 2014;9:e96486.

12. Cheeseman $\mathrm{IH}$, Miller BA, Nair S, Nkhoma S, Tan A, Tan JC, et al. A major genome region underlying artemisinin resistance in malaria. Science. 2012;336:79-82.
13. Schlötterer C. Evolutionary dynamics of microsatellite DNA. Chromosoma. 2000;109:365-71.

14. Wootton JC, Feng X, Ferdig MT, Cooper RA, Mu J, Baruch DI, et al. Genetic diversity and chloroquine selective sweeps in Plasmodium falciparum. Nature. 2002;418:320-3.

15. Cheeseman IH, Miller BA, Nair S, Nkhoma S, Tan A, Tan JC, et al. A major genome region underlying artemisinin resistance in malaria. Science. 2012;336:79-82.

16. Amambua-Ngwa A, Tetteh KKA, Manske M, Gomez-Escobar N, Stewart LB, Deerhake ME, et al. Population genomic scan for candidate signatures of balancing selection to guide antigen characterization in malaria parasites. PLoS Genet. 2012;8:e1002992.

17. Kim Y, Neilsen R. Linkage disequilibrium as a signature of selective sweeps. Genetics. 2004;167:1513-24.

18. Sabeti PC, Reich DE, Higgins JM, Levine HZP, Richter DJ, Schaffner SF, et al. Detecting recent positive selection in the human genome from haplotype structure. Nature. 2002;419:832-7.

19. Jensen JD, Kim Y, DuMont VB, Aquadro CF, Bustamante CD. Distinguishing between selective sweeps and demography using DNA polymorphism data. Genetics. 2005;170:1401-10.

20. Dittrich S, Mitchell SL, Blagborough AM, Wang Q, Wang P, Sims PFG, et al. An atypical orthologue of 6-pyruvoyltetrahydropterin synthase can provide the missing link in the folate biosynthesis pathway of malaria parasites. Mol Microbiol. 2008;67:609-18.

21. Farooqui AA, Horrocks LA. Excitatory amino acid receptors, neural membrane phospholipid metabolism and neurological disorders. Brain Res Brain Res Rev. 1991;16:171-91.

22. Zidovetzki R, Sherman IW, O'Brien L. Inhibition of Plasmodium falciparum phospholipase A2 by chloroquine, quinine, and arteether. J Parasitol. 1993;79:565-70.

23. Pappa V, Mony V, Rhee K, Daily J. Phospholipase A2 inhibition in Plasmodium falciparum: a potential novel antimalarial strategy. Am J Trop Med Hyg. 2014;Abstracts:86-7.

24. Cairns M, Roca-Feltrer A, Garske T, Wilson AL, Diallo D, Milligan PJ, et al. Estimating the potential public health impact of seasonal malaria chemoprevention in African children. Nat Comm. 2012;3:881.

25. Schlitzer M. Malaria chemotherapeutics part I: history of antimalarial drug development, currently used therapeutics, and drugs in clinical development. ChemMedChem. 2007;2:944-86.

26. Drakeley CJ, Akim NI, Sauerwein RW, Greenwood BM, Targett GA. Estimates of the infectious reservoir of Plasmodium falciparum malaria in The Gambia and in Tanzania. Trans R Soc Trop Med Hyg. 2000;94:472-6.

27. Ceesay SJ, Casals-Pascual C, Nwakanma DC, Walther M, Gomez-Escobar $\mathrm{N}$, Fulford AJC, et al. Continued decline of malaria in The Gambia with implications for elimination. PLoS ONE. 2010;5:e12242.

28. Manske M, Miotto O, Campino S, Auburn S, Almagro-Garcia J, Maslen G, et al. Analysis of Plasmodium falciparum diversity in natural infections by deep sequencing. Nature. 2012;487:375-9.

29. Benson G. Tandem repeats finder: a program to analyze DNA sequences. Nucleic Acids Res. 1999;27:573-80.

30. Kofler R, Schlotterer C, Lelley T. SciRoKo: a new tool for whole genome microsatellite search and investigation. Bioinformatics. 2007;23:1683-5.

31. Highnam G, Franck C, Martin A, Stephens C, Puthige A, Mittelman D. Accurate human microsatellite genotypes from high-throughput resequencing data using informed error profiles. Nucleic Acids Res. 2013;41:e32.

32. Tae H, Kim DY, McCormick J, Settlage RE, Garner HR. Discretized Gaussian mixture for genotyping of microsatellite loci containing homopolymer runs. Bioinformatics. 2014;30:652-9.

33. Dieringer D, Nolte V, Schlötterer C. Population structure in African Drosophila melanogaster revealed by microsatellite analysis. Mol Ecol. 2005;14:563-73. 